\title{
DEGRADAÇÃO DE ACIDO ASCÓRBICO EM PRESENÇA DE OLIGOELEMENTOS EM NUTRIÇÃO PARENTERAL
}

\section{DEGRADATION OF ASCORBIC ACID IN THE PRESENCE OF TRACE ELEMENTS IN PARENTERAL NUTRITION}

Éder Luiz OTTO'; João Willian CHASTE²; Larissa COMARELLA ${ }^{3}$; Astrid WIENS ${ }^{4}$; Andréia Cristina Conegero SANCHES ${ }^{5}$; Marco André CARDOSO ${ }^{6}$; Claudete JACYCZEN ${ }^{7}$; Roberto PONTAROLO ${ }^{8}$

1 - Farmacêutico assistente técnico da Central de Manipulação de Quimioterapia Nutrição Enteral e Parenteral.

2-Farmacêutico Técnico Responsável da Rochafarma Farmácia e Perfumaria Ltda.

3 - Mestre em Ciências (Bioquímica) - Professora do Departamento de Farmácia - Centro Universitário Campos de Andrade

4 - Doutoranda do Programa de Pós-Graduação em Ciências Farmacêuticas - Universidade Federal do Paraná

5 - Farmacêutica, docente do curso de Farmácia da Universidade Estadual do Oeste do Paraná UNIOESTE

6 - Doutor em Bioquímica pela Universidade Federal do Paraná.

7 - Farmacêutica especialista em Nutrição Enteral e Parenteral.

8 - Doutor em Bioquímica; Professor do Departamento de Farmácia - Universidade Federal do Paraná.

\section{RESUMO:}

A nutrição parenteral (NP) consiste na administração das exigências nutricionais diárias. A mistura é complexa e composta por muitas substâncias, entre elas o ácido ascórbico (AA) e o cobre, que podem interagir na presença de oxigênio. Essa pesquisa teve como objetivo verificar se essa interação ocorre na presença de todos os componentes da NP quando adicionados na mesma bolsa. Para a realização dos ensaios foram utilizadas seis bolsas padrão de NP, sendo que três delas não continham a solução de oligoelementos. Todas foram deixadas $24 \mathrm{~h}$ na geladeira e mais $24 \mathrm{~h}$ à temperatura ambiente. Para verificar a degradação do AA, foi utilizada metodologia descrita por GIBBONS e colaboradores (2001) com detecção da degradação do AA por HPLC. Observou-se variação da concentração do AA total em função do tempo. Após retirar as bolsas da geladeira houve uma queda acentuada da concentração do AA nas seis bolsas. A diferença da concentração do AA nas bolsas com ou sem oligoelementos (inclusive cobre) não foi significativa. Portanto, a degradação do AA parece ocorrer da mesma forma em bolsas contendo ou não oligoe- 
elementos. No entanto, como existe uma rápida degradação do AA em temperatura ambiente, seria vantajoso do ponto de vista clínico, que a NP fosse produzida em duas bolsas, adicionando o AA somente na primeira delas e o cobre na segunda bolsa, pois a infusão da bolsa 1 ocorre em apenas 12 horas, o que leva o paciente a receber maior quantidade desse componente.

Palavras-chave: nutrição parenteral; interações; ácido ascórbico; cobre.

\section{ABSTRACT:}

Parenteral nutrition (PN) consists of the administration of the daily nutritional requirements. The mixture is complex and composed of many substances, including ascorbic acid (AA) and copper, which can interact in the presence of oxygen. This study aimed to determine whether this interaction occurs in the presence of all components of the PN when added in the same bag. For the tests we used six bags with standard PN, three of which contained no trace elements (including copper) in the solution. The bags were left 24 hours in the refrigerator and an additional 24 hours at room temperature. To check the degradation of AA, we used the method described by GIBBONS and colleagues (2001), detection of the degradation of AA by HPLC. The concentration of total $A A$ varied as a function of time. After removing the bags from the refrigerator there was a lower concentration of $A A$ in the six bags. The difference in concentration of $A A$ on the bag with or without trace elements (including copper) was not significant. Therefore, the degradation of AA seems to occur in the same way in bags with or without trace elements. However, as there is a rapid degradation of AA at room temperature, it would be advantageous from a clinical standpoint, to produce the PN in two bags, adding only $A A$ in the first and copper in the seccond, because the infusion bag 1 takes place in only 12 hours, which causes the patient to receive the greatest amount of this component.

Keywords: parenteral nutrition; interactions; ascorbic acid; copper.

\section{INTRODUÇÃO}

O suporte nutricional parenteral consiste na administração de todas, ou parte das exigências nutricionais diárias, e está indicado em casos em que os pacientes não podem, não devem e não querem se alimentar por via gastrintestinal (MARCHINI et al., 1998), podendo ser devido a vários fatores, como antes e após cirurgias do trato gastrintestinal, desordens gastrintestinais e condições pediátricas (prematuros) (WAITZBERG, 2004).

A nutrição parenteral é complexa e composta por muitas substâncias: água, carboidratos, proteínas, lipídios, eletrólitos, vitaminas e minerais, por isso deve-se tomar cuidado com alguns aspectos, principalmente devido a incompatibilidades físico- 
químicas da solução ou emulsão (SCHRODER, 2008). Entre estas estão peroxidação lipídica (em emulsões), floculação, preciptação e inativação de substâncias devido a instabilidades químicas entre os componentes como, por exemplo, pode ocorrer oxidação da vitamina $C$, ou ácido ascórbico (AA) presente na solução de polivitaminas, catalisada por íons metálicos, especialmente o cobre, que está presente na solução de oligoelementos. Alguns autores afirmam que por questões físicas e químicas esses dois componentes não podem ser adicionados na mesma bolsa, uma vez que o AA sofre degradação (oxidação) na presença de oxigênio e de cobre, diminuindo o valor nutricional da terapia parenteral (SCHRODER, 2008, TRISSEL, 1994 GIBBONS, ALLWOOD \& HARDY).

O AA é uma vitamina hidrossolúvel, de estrutura simples cuja oxidação leva a formação do ácido dihidroxiascórbico (DHAA) e este pode ser posteriormente hidrolisado a ácido 2,3-dicetogulônico. Destes, apenas os ácidos ascórbico e dihidroxiascórbico, possuem atividade biológica. A conversão de AA para DHAA leva à condições anaeróbicas na bolsa de NP, já que o oxigênio é utilizado na oxidação. Essa primeira reação pode ser revertida utilizando-se um forte agente redutor, como o ditiotreitol (DTT). A hidrólise do DHAA leva a formação de um produto inativo, sem função na nutrição e cuja reação reversa não ocorre (como acontece no caso do AA e DHAA). Essa última reação é independente de cobre, ou qualquer outro oligoelemento, mas depende diretamente da temperatura de armazenamento e do $\mathrm{pH}$ (BODE, CUNNINGHAM \& ROSE, 1990; GIBBONS, ALLWOOD \& HARDY 2001). Se armazenado refrigerado, em pH 3,0 a 5,0 essa hidrólise ocorre em baixa quantidade e temperaturas acima de $15^{\circ} \mathrm{C}$ a $30^{\circ} \mathrm{C}$ e pH 6,0 a 8,0 aceleram essa degradação (BODE, CUNNINGHAM \& ROSE, 1990; GIBBONS, ALLWOOD \& HARDY 2001).

No entanto, apesar da bolsa ser mantida refrigerada antes do uso, a administração da NP é realizada em temperatura ambiente, sendo impossível evitar a hidrólise do DHAA. Dessa forma busca-se evitar a oxidação do AA, o que aparentemente é viável se não houver oxigênio e oligoelementos na solução. Porém deve-se levar em consideração que não há como evitar o contato da solução com o oxigênio, que ocorre tanto na manipulação como na administração da NP.

Para tal, em alguns hospitais ou laboratórios de manipulação, a NP é manipulada em duas bolsas separadas, sendo que uma contém as substâncias polivitamínicas e a outra oligoelementos. No entanto, isso aumenta o custo da NP, além de aumentar os riscos de contaminações para o paciente, que precisa utilizar duas bolsas por dia, ao invés de uma só.

Devido às controvérsias que ainda existentem com relação à mistura desses componentes na mesma bolsa, há necessidade de estudos voltados à estabilidade da vitamina $\mathrm{C}$, quando esta etiver associada com oligoelementos, na presença de todos os 
macro e micronutrientes que são usualmente utilizados em NP. Se comprovado que a degradação do AA na temperatura e tempo de armazenamento da NP for baixa, e que não levará a deficiência no fornecimento de vitamina $C$ ao paciente, então, estas substâncias poderão ser adicionadas na mesma bolsa. Mas, se verificado aumento da degradação do $A A$, chegando a níveis de vitamina $C$ abaixo do recomendado, então é ideal que a NP seja manipulada em dois planos, separando os dois compostos.

Um estudo realizado por Alwood, em 1984, avaliou a quantidade de AA degradado na presença de oxigênio, comparando soluções contendo cobre ou não. Na ausência de cobre, a degradação do AA foi de 20 a 30mg após 24 horas do preparo da $\mathrm{NP}$, enquanto que na presença de cobre essa degradação aumentou para 150 a $200 \mathrm{mg}$ somente duas horas após a manipulação. Nesse estudo os autores concluíram que o cobre e o AA não devem ser utilizados na mesma bolsa. No entanto, os componentes foram adicionados à água, e não à uma solução completa de NP (ALWOOD, 1984). Em outro estudo, PROOT e colaboradores (1994) também verificaram diminuição nos níveis de AA quando adicionado a soluções de NP contendo oligoelementos. No entanto a maior degradação ocorreu devido à presença de oxigênio, e não à interação entre os dois componentes.

A partir destes dados, essa pesquisa objetivou verificar e confirmar se na NP, a presença de oligoelementos leva a degradação significativa de AA. Este trabalho pretende contribuir com futuras pesquisas e desenvolvimentos nesta área de NP, para que, se for verificado pouca redução na concentração de vitamina $C$, o tratamento possa a ser realizado utilizando apenas uma bolsa, reduzindo assim os riscos de contaminação ao paciente no momento da troca da bolsa, bem como os custos da terapia parenteral. Com esta pesquisa, pretendeu-se verificar os níveis de degradação doAA, em função do tempo, na presença e ausência de oligoelementos.

\section{MATERIALE MÉTODOS}

Este trabalho foi aprovado pelo Comitê de Ética em Pesquisa em Seres Humanos, do Centro Universitário Campos de Andrade, sob o número de protocolo 000376. Os ensaios foram realizados no laboratório da Universidade Federal Paraná Curitiba e foram utilizados reagentes e soluções fornecidas pela empresa CEQNEP de Curitiba, (Central de Manipulação de Quimioterapia Nutrição Enteral e Parenteral). Para verificar a degradação do AA, foi utilizada metodologia descrita por GIBBONS e colaboradores (2001) com detecção da degradação por HPLC (High Pressure Liquid Cromathography). Os ensaios foram realizados em triplicata.

Preparo das bolsas de NP

Visão Acadêmica, Curitiba, v.12, n.2, Jul. - Dez./2011 - ISSN 1518-5192 
Para a realização dos ensaios, visando detectar a concentração de AA em função do tempo, foram utilizadas seis bolsas padrão de NP, sendo que, três delas não continham a solução de oligoelementos e as outras três bolsas, continham a solução de oligoelementos associada a solução de polivitamina. As bolsas utilizadas foram do tipo EVA (Etileno Acetato de Vinila) e técnicas assépticas foram utilizadas de forma a minimizar a contaminação microbiana da mistura de nutrientes.

As bolsas foram manipuladas em temperatura em torno de $18^{\circ} \mathrm{C}$, fluxo horizontal, classe ISO 5, 100 partículas por pés ${ }^{3}$ de ar, em sala limpa Classe ISO 7, 10.000 partículas por pés ${ }^{3}$ de ar e, a estas, foram adicionados todos os componentes da NP, com exceção da solução de polivitamina (ampola A, contendo a vitamina C). As bolsas de NP foram preparadas no CEQNEP e transportadas em caixa térmica refrigerada $\left(2\right.$ a $\left.8^{\circ} \mathrm{C}\right)$ à UFPR, onde os ensaios em HPLC foram realizados. A solução de polivitaminas foi adicionada a todas as bolsas, aproximadamente 1 hora após o preparo destas no CEQNEP.

Como se desconhece o pico do DHAA, seguiu-se a metodologia descrita por GIBBONS e colaboradores (2001), adicionando DTT 0,008mol/L a cada vial, 15 minutos antes de cada leitura. Portanto, a cada retirada de amostra de 0,5 ml, foram adicionados $0,5 \mathrm{ml}$ de solução de DTT diretamente no vial repouso durante 15 minutos, protegido da luz. Todas as amostras foram filtradas em filtro Millex® 0,22 micra. As bolsas permaneceram ao abrigo da luz e em temperatura de $5-7^{\circ} \mathrm{C}$ durante os tempos 0 às $24 \mathrm{~h}$ e $21,3-21,9^{\circ} \mathrm{C}$ entre 24 às $48 \mathrm{~h}$. As leituras foram realizadas em nos tempos: $\mathrm{t}$ $=0 \mathrm{~h}, 2 \mathrm{~h}, 4 \mathrm{~h}, 6 \mathrm{~h}, 12 \mathrm{~h}, 24 \mathrm{~h}, 36 \mathrm{~h}$ e $48 \mathrm{~h})$.

\section{RESULTADOS E DISCUSSÃO}

\section{Determinação da degradação do $A A$}

Para verificar a extenção de degradação do AA na presença ou ausência de oligoelementos, seis bolsas de NP foram preparadas, sendo que, um jogo de triplicata continha a solução de oligoelementos misturada com a de polivitaminas (ampola A, que contém a vitamina $C$ ), enquanto que as outras três continham apenas polivitaminas, sem a presença de oligoelementos. A figura 1 mostra a variação, em porcentagem, da concentração do AA total em função do tempo. 


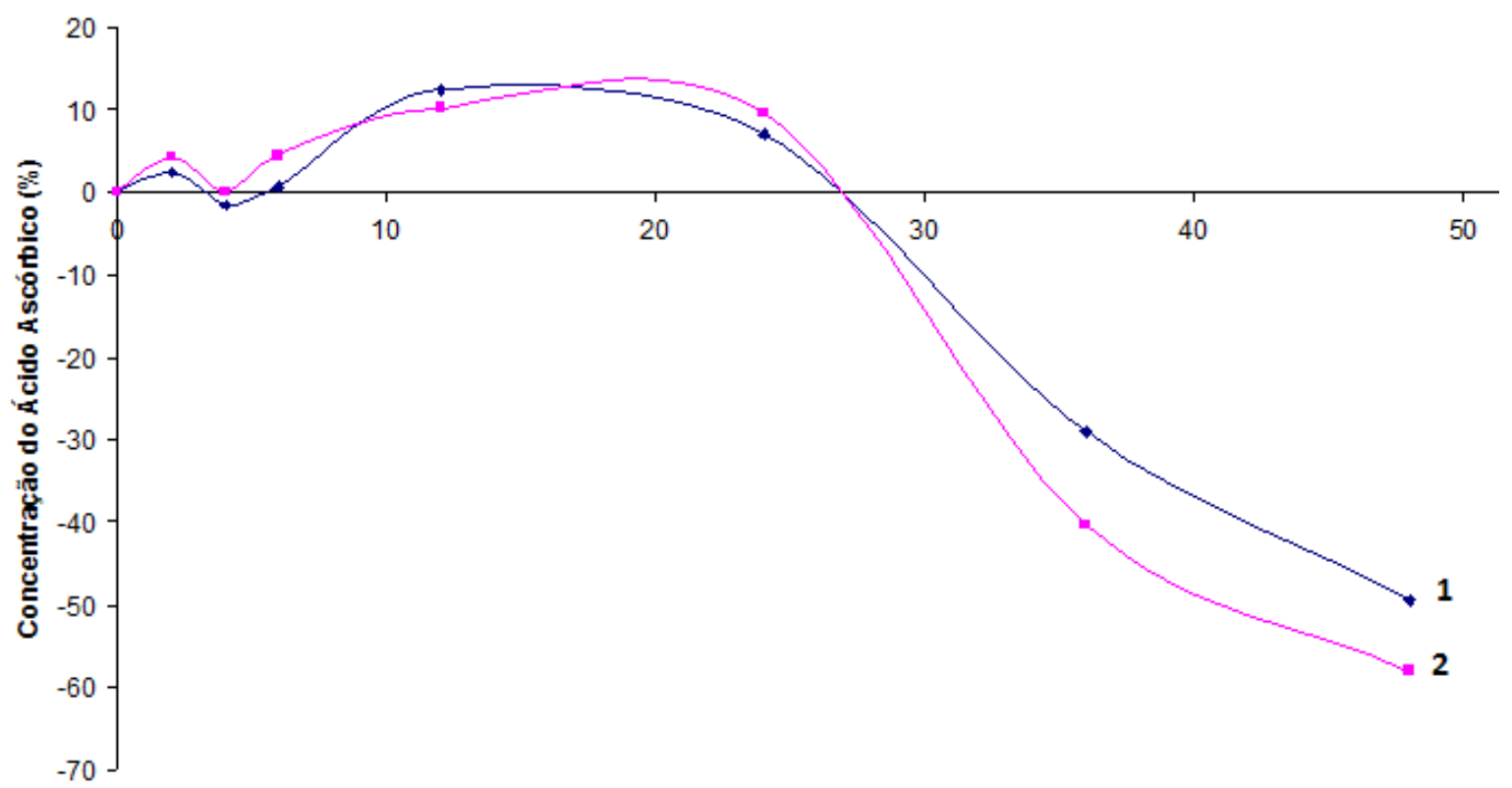

Tempo (horas)

Figura 1: Variação da degradação do AA na mistura de np em função do tempo. (1) - bolsa NP contendo Polivitaminas ; (2) - bolsa NP contendo Polivitaminas + Oligoelementos associados. Eixo vertical: degradação do AAa ácido 2,3 dicetogulônico (\%); Eixo horizontal: tempo (horas).

Neste trabalho, padronizou-se por degradação final do AA, a sua conversão no produto biologicamente inativo, o ácido 2,3-dicetogulônico, não importando a quantidade de DHAA formado durante o ensaio, uma vez que este, além de possuir atividade, na presença do DTT, reduz-se a AA original.

A partir dos dados apresentados na figura 1, pode-se observar que, do tempo 0h até $6 \mathrm{~h}$, a concentração de AA sofre poucas variações, indicando que, nesta etapa, ocorre pouca degradação a ácido 2,3-dicetogulônico. Esse resultado não significa que o AA não se oxide a DHAA, porém este subproduto não foi mensurado durante o experimento, pois não há pico de detecção deste por HPLC. Como antes de cada leitura, o DTT foi adicionado à amostra e aguardando-se 15 minutos até análise, neste tempo ele reagiu com as moléculas de DHAA presentes no meio, convertendo-as à AA novamente. Portanto, determina-se como concentração de AA a soma deste com o DHAA, resultando no que consideramos como AA total ou AAT. Este tempo inicial (0 a $6 \mathrm{~h})$ mostrou que, se armazenada sob refrigeração $\left(5\right.$ a $\left.7^{\circ} \mathrm{C}\right)$, a vitamina $C$ (AAT) sofre pouca variação na sua concentração.

No $t=12 \mathrm{~h}\left(5\right.$ a $\left.7^{\circ} \mathrm{C}\right)$ e nesta, observou-se uma pequena elevação na concentração do AAT, resultado inesperado por se tratar de um estudo de degradação. Este resultado pode ter algumas justificativas, entre elas a possibilidade de uma com- 
plexação da vitamina C com outros componentes da NP e que tenham impedido a leitura correta desta pelo cromatógrafo, no início do ensaio (entre 0 e $6 \mathrm{~h}$ ).

A próxima leitura foi realizada passadas 24 horas do preparo da mistura. Geralmente nos hospitais, decorridas 24 horas pós fabricação, a bolsa de NP é retirada da refrigeração e encaminhada a algum paciente em tratamento, o qual pode receber os nutrientes da bolsa em tempos que variam de poucas horas até 1 dia, dependendo do caso. Por isso, a partir desta etapa, as bolsas foram mantidas à temperatura ambiente (em torno de $22^{\circ} \mathrm{C}$ ), para realização dos ensaios subsequentes. No $\mathrm{t}=24 \mathrm{~h}$ começou a se evidenciar uma redução na concentração de AAT, ainda não tão brusca.

Após 36 horas, observou-se uma significativa queda na concentração de AAT. No caso da mistura contendo oligoelementos, essa redução foi de $40 \%$, enquanto que, nas bolsas sem oligo a redução foi de $28 \%$, um pouco menor. Na próxima análise, que foi a do $t=48 \mathrm{~h}$, a degradação da vitamina $\mathrm{C}$ chegou em torno de $50 \%$, em ambos os casos.

Sabe-se que a temperatura influencia na velocidade de degradação do DHAAa ácido 2,3-dicetogulônico e, este resultado foi claramente evidenciado neste experimento, onde foi possível verificar redução acentuada da vitamina $\mathrm{C}$, quando a bolsa de NP é deixada à temperatura ambiente. Gibbons e colaboradores (2001) já haviam demonstrado que a degradação do AA é mais dependente da temperatura do que da presença de oligoelementos. Em seus experimentos, verificaram que baixas temperaturas $\left(0\right.$ a $\left.15^{\circ} \mathrm{C}\right)$ mantinham o DHAA estável, porém o aumento desta (a partir de $15^{\circ} \mathrm{C}$ ) e em meio anaeróbico, estimulava a hidrólise ao produto inativo. Além da temperatura, deve-se considerar $\mathrm{o} \mathrm{pH}$ da mistura, uma vez que já foi descrito a estabilidade do DHAA em pH ácido (3,0-5,0) (BODE, CUNNINGHAM \& ROSE, 1990). Um aumento do $\mathrm{pH}$ na mistura pode ocorrer devido a instabilidades físico-químicas dentro do sistema, o qual é rico em diferentes substâncias e assim, acelerar a degradação do DHAA.

Na maioria dos hospitais, as bolsas de NP ficam armazenadas sob refrigeração por até 24 horas, após este período, obrigatoriamente devem ser encaminhadas ao paciente, pois a vida útil de uma bolsa é de 48 horas (por isso os ensaios foram realizados de 0 a 48h). Como a infusão da NP ocorre continuamente, durante 24 horas, a bolsa fica sob temperatura ambiente durante todo esse período.

Conforme verificamos através dos experimentos, a partir do momento que a bolsa é retirada da refrigeração, a velocidade de degradação da vitamina $\mathrm{C}$ aumenta e, se há presença de oxigênio e catalisadores na bolsa, podemos extrapolar este resultado para qualquer tempo, seja $2 \mathrm{~h}, 6 \mathrm{~h}$ ou $12 \mathrm{~h}$. Se a temperatura aumentar, e na bolsa já houver altas concentrações de DHAA (proveniente da oxidação do AA), a hidrólise deste composto é acelerada e, há redução da concentração da vitamina $C$ biologicamente ativa na mistura. A degradação mostrou-se diretamente independente 
da presença de oligoelementos, visto que as concentrações das diferentes bolsas (com ou sem oligoelementos) apresentraram comportamento e decréscimo similar. Agrande interferência dos íons metálicos (oligoelementos) seria acelerando o processo de conversão do AA em DHAA e, posterior degradação deste influenciado pela temperatura.

Acredita-se, portanto, que o ideal seria manter na mistura de NP, uma concentração maior de AA em relação ao DHAA, pois a oxidação daquele não sofre interferência da variação de temperatura. Por isso, ao reduzir ao máximo as concentrações de oxigênio e aeração do sitema, impede-se que, quantidade considerável do AA se oxide a DHAA, otimizando o tempo de armazenamento da mistura, uma vez que o primeiro é mais estável que seu produto de oxidação, como já verificado anteriormente (GIBBONS, ALLWOOD \& HARDY 2001).

A principal causa de perda de AA em misturas de NP é a sua reação com o oxigênio, que pode estar presente na bolsa por diversos meios, como: proveniente de reações entre os componentes, do processo de preparo das bolsas, das seringas de coleta, bem como das bolsas plásticas que permitem a passagem do oxigênio do meio externo para o interno, sendo esta última fonte minimizada pela utilização de bolsas trilaminadas. Reduzindo-se, portanto a concentração do oxigênio previne-se a maior oxidação da vitamina C e aumento do subproduto ativo DHAA. Quando a bolsa, contendo pequenas quantidades de DHAA estiver em temperatura ambiente, apenas baixas concentrações de ácido 2,3-dicetogulônico serão produzidas e uma maior quantidade de vitamina $\mathrm{C}$, na forma de $\mathrm{AA}$ ativo, fica disponível ao paciente, maximizando a função da terapia por NP que é a de fornecer os nutrientes necessários ao corpo, nas quantidades recomendadas.

\section{CONCLUSÕES}

Conclui-se que, a degradação do AA depende diretamente da variação da temperatura. Nesse trabalho não foi encontrada relação da presença ou ausência de oligoelementos na degradação do AA.

Portanto, a degradação da vitamina C parece ocorrer da mesma forma em bolsas contendo oligoelementos e em bolsas sem a presença desses oligoelementos. No entanto, como existe uma rápida degradação da vitamina $C$ em temperatura ambiente, seria vantajoso, do ponto de vista clínico, que a bolsa de NP continuasse sendo produzida em duas bolsas, adicionando vitamina $\mathrm{C}$ somente na primeira delas, pois assim o paciente receberia uma maior concentração de vitamina $\mathrm{C}$, pois a infusão da bolsa 1 ocorre em apenas 12 horas.

Apesar do tratamento em bolsas separadas ser mais complicado devido aos 
elevados custos e riscos associados, deve-se prevalecer a idéia de que, a terapia de NP é a única forma pela qual alguns pacientes podem receber os elementos necessários à sua sobrevivência e, a redução na concentração destes nutrientes, podem trazer resultados negativos ao tratamento.

\section{REFERÊNCIAS}

ALLWOOD, M.C. Factors influencing the stability of ascorbic acid in total parenteral nutrition infusions. J Clin Hosp Pharm, n. 9, v. 2, p. 75-85, 1984.

BERTOL, C.D.; ADAMS, A.I.H; WERLANG, M.C. Procedimento para Validação do Processo de Preparação de NP. Rev Bras Nutr Clin, n. 21, v. 3, p. 193-197, 2006.

BODE, A.M.; CUNNINGHAM, L.; ROSE, R.C. Spontaneous decay of oxidized ascorbic acid (dehydro - L - ascorbic acid) evaluated by High-Pressure Liquid Chromatography. Clin. Chem. v. 36, n.10, p. 1807-1809, 1990.

BRASIL. Ministério da Saúde. Portaria $n^{0}$ 272, de 08 de abril de 199. Aprova o Regulamento Técnico para fixar os requisitos mínimos exigidos para a Terapia de NP. Diário Oficial da União [da República Federativa do Brasil], Brasília, 1998.

CAMPANELLA, L.C.A.; SILVEIRA, B.M.; NETO, O.R.; SILVA, A.A. Terapia Nutricional enteral: a dieta prescrita é realmente infundida? Rev Bras Nutr Clin, n. 23, v. 1, p. 21$25,2008$.

GIBBONS, E., ALLWOOD, M.C., NEAL, T. e HARDY, G. Degradation of dehydroascorbic acid in parenteral nutrition mixtures. J Pharm Biomed Anal, n. 25, v. 3-4, p. 605-11, 2001.

KRAUSE, E.S., MAHAN, S., KATHLEEN, L. .Alimentos, nutrição e dietoterapia. Roca: São Paulo, $9^{a}$ ed. 1998.

MARCHINI, J.S., OKANO, N.; CUPO, P.; PASSOS, N.M.R. da S. NP - Príncipios Gerais, Formulários de Prescrição e Monitorização. Nutr Clin, n. 31, p. 62-72, 1998.

PROOT P, DE POURCQ L, RAYMAKERS AA. Stability of ascorbic acid in a standard total parenteral nutrition mixture. Clin Nutr, n. 13, v. 5, p. 273-9, 1994. 
SCHRODER, A.M. Total Parenteral Nutrition - Problems in Compatibility and Stability. J. Eur Hosp Pharm. v 14, p. 65-67, 2008/1.

Eur Hosp Pharm. v 14, p. 65-67, 2008/1.

TRISSEL, A. Handbook on Injectable Drugs by Lawrence edition 1994.

WAITZBERG, Dan L. Nutrição oral, enteral e parenteral na prática clínica. ed.3 Vol. 1. Atheneu. São Paulo- SP, 2002. 